University of Washington, Seattle: Johns Hopkins University Hospital, Baltimore: and University of Pittsburgh School of Medicine, PA. Head circumference was also measured. Children with autism 12 years of age and younger had significantly larger brain volumes (average $5 \%$ increase) compared to normal children. Autism and control groups older than age 12 years showed no differences in brain volume. The accelerated growth in brain volume and increased head circumference noted in younger children with autism was slowed to an average of 1 to $2 \%$ increase in older children. Brain volume in adolescents and adults with autism appears to show a slight decrease while that of normal controls continues to increase. (Aylward EH, Minshew NJ, Field K, Sparks BS, Singh N. Effects of age on brain volume and head circumference in autism. Neurology July (2 of 2) 2002;59:175-183). (Reprints: Dr Elizabeth H Aylward, Department of Radiology, Box 357115, University of Washington School of Medicine, Seattle, WA 98195).

COMMENT. Autism is a spectrum of developmentai disorders, grouped under the term 'pervasive developmental disorder' (PDD), with impairments of social interaction, communication, and repetitive or restricted behaviors. While autistic disorder is characterized by all three behavior patterns, Asperger's syndrome is milder than autistic disorder and is distinguished by normal language development. Within these categories the clinical manifestations show wide heterogeneity. The cause of autism is unknown and the clinical variation in the degrees of autistic spectrum disorder complicate the evaluation of neuroimaging studies. As pointed out by Mink JW and McKinstry RC, in their editorial (Neurology 2002;59:158-159), despite some contradictory results, imaging studies may provide a noninvasive in vivo mathod of investigating the neurobiology of autism.

The above study by Aylward and associates and that of Sparks et al, in the same issue (Neurology 2002;59:184-192), found increases in brain volume of young children with autism. Sparks also found that cerebellar, amygdalar, and hippocampal volumes increased in proportion to growth of the cerebral volume of autistic children. The average cerebral volume is 5 to $10 \%$ larger in autistic compared to normal children. After the age of 12 years, brain growth shows a plateau, and brain size in adolescents and adults is similar in autistic and control patients. Future studies will require careful selection of subjects and measurement of the growth of specific brain regions. The neurobiological and functional significance of these structural changes in the brain remains to be determined.

\title{
PRACTICAL RELEVANCE OF MRI IN DIAGNOSIS OF AUTISM AND ADHD
}

Methods of quantitative MRI brain mapping and possible significance in diagnosis and response to therapy of childhood autism and attention deficit hyperactivity disorder are reviewed by investigators at the Child Psychiatry Branch, National Institute of Mental Health, Bethesda, MD. Recent advances in MRI allow automated analysis in measurement of regional gray and white matter volumes across time and between subject groups. Standard curves of healthy and disease-specific regional maturation and volumetric changes during development may be helpful in diagnosis. Except for the cerebellum, volume measurements are more highly correlated for monozygotic than dizygotic twins, and heritability indices for brain regions may be calculated. Brain volume and region-specific brain tissue changes may identify patients at high risk for autistic disorder and ADHD. 
In studies at the NIH, childhood-onset schizophrenia is characterized by region-specific progressive cortical gray matter loss in parietal, frontal, and temporal regions. A "wave" of back-to-front tissue loss, early parietal followed by frontal and temporal gray matter loss, occurs in adolescence. Loss of gray matter slows in early adulthood. In contrast, two large studies of 57 boys and 50 girls with ADHD showed smaller brain size, abnormal caudate, and decreased volume of the posterior inferior cerebellar vermis, when compared to 105 matched controls. Anatomical changes in patients with ADHD support a postulated dysfunction of cerebellar-striatal-prefrontal brain circuits. Although results of MRI studies at present are promising, they are not sufficiently homogeneous to indicate routine brain MRI in childhood neuropsychiatric disorders. (Gogtay N, Giedd J, Rapoport JL. Brain development in healthy, hyperactive, and psychotic children. Arch Neurol August 2002;59:1244-1248). (Reprints: Judith L Rapoport MD, Child Psychiatry Branch, National Institute of Mental Health, National Institutes of Health, Bldg 10, Rm 3N202, 10 Center Dr, MSC 1600, Bethesda, MD 20892).

COMMENT. These large prospective MRI studies of the brains of hyperactive, autistic, and healthy children and adolescents, undertaken at the NIH since 1990, have uncovered subtle structural changes in brain development that supplement postmortem findings and are potentially significant in diagnosis and management.

Impaired activation of prefrontal-temporal regions demonstrated by PET studies in autistic subjects at the Wellcome Department of Cognitive Neurology, London, UK (Castelli F, Frith C, Happe F, Frith U. Autism, Asperger's syndrome and brain mechanisms for the attribution of mental states to animated shapes. Brain August 2002;125:1839-1849). While watching animated sequencies depicting two triangles moving interactively, a 'mentalizing' effect that is normally associated with specific brain activation is impaired in autistic compared to control subjects. It is theorized that autism is the result of impaired mentalizing, and is characterized by a lack of social insight and poor communicative skills (Baron-Cohen et al. 1985).

\section{HEADACHE DISORDERS}

\section{PRACTICE PARAMETERS FOR EVALUATION OF HEADACHE}

The Quality Standards Subcommittee of the American Academy of Neurology and the Practice Committee of the Child Neurology Society have developed practice parameters for evaluation of children and adolescents with recurrent headaches. Individual committee members reviewed titles and abstracts for content and relevance. Articles selected included studies with more than 25 patients, those with details of a neurologic examination, and those concerning etiology. Of those reviewed, only one study reported results of laboratory tests and none concerned the role of routine lumbar puncture. Eight studies assessed the use of EEG in 1,148 children with recurrent headache, but none compared the incidence of EEG abnormalities in migraine vs nonmigraine pediatric headache patients.

EEG was not recommended for routine evaluation, even in patients suspected of having seizure-related headaches. Neuroimaging should only be considered in patients with an abnormal neurologic examination or findings suggesting CNS disease. Predictors of a space-occupying lesion included: 1) recent-onset or change in type of severe headaches; 2) absent family history of migraine; 3) abnormal neurologic exam; 4) gait abnormalities; and 5) seizures. 